\title{
Prevalence of NAFLD and Its Correlation with Diabetic Nephropathy in Type 2 Diabetes Mellitus Patients
}

\author{
Simran Kaur ${ }^{1}$, Sushma Laxmareddygari² ${ }^{2}$ Nitin Gupta ${ }^{3}$, Akshith Rajgupta Tabjula ${ }^{4}$, Yavatesh Joshi ${ }^{5}$, Sargam Dhaliwal ${ }^{6}$
}

1,2.3, 4, 5, 6 Department of Medicine, M.M. Institute of Medical Sciences and Research, Maharishi Markandeshwar (Deemed to Be University), Mullana, Haryana, India.

\section{ABSTRACT}

\section{BACKGROUND}

Diabetes mellitus (DM) is a disease related to abnormality in metabolism of carbohydrates which is characterised by hyperglycemia that results from relative or absolute defect in insulin secretion, varying degrees of insulin resistance or both. Various researches have established the link between type 2 diabetes mellitus (T2DM) and non-alcoholic fatty liver disease (NAFLD). The present study was done for assessing the prevalence of NAFLD and its association with diabetic nephropathy in patients with type 2 diabetes mellitus.

\section{METHODS}

75 patients of type 2 diabetes were included in this study. Clinical details and demographic data of all the patients were obtained. Samples were obtained from all the patients and all the routine investigations were carried out. Ultrasonography (USG) abdomen was performed in all patients after 12 hours fasting. Microsoft Excel was used for recording the results and were analysed by Statistical Package for the Social Sciences (SPSS) software. Level of significance was evaluated using chisquare test.

\section{RESULTS}

NAFLD was present in 57 patients. Therefore, the overall prevalence of NAFLD was see $76 \%$. The overall prevalence of diabetic nephropathy was 42 percent among diabetic patients. Out of 31 patients with diabetic nephropathy, NAFLD was present in 25 patients. Significant results were obtained while assessing the association of NAFLD with diabetic nephropathy in T2DM patients.

\section{CONCLUSIONS}

NAFLD was found to be a significant risk factor for the development of diabetic nephropathy in patients having type 2 diabetes mellitus.

\section{KEY WORDS}

Non-Alcoholic Fatty Liver, Diabetic Nephropathy, Type 2 Diabetes Mellitus, Nonalcoholic Steatohepatitis
Corresponding Author:

Dr. Nitin Gupta,

Associate Professor,

Department of General Medicine,

Maharishi Markandeshwar Institute of Medical Sciences \& Research (Maharishi Markandeshwar Deemed to Be University)

Mullana, Ambala, Haryana, India

E-mail: drnitintayal@gmail.com

DOI: $10.14260 /$ jemds/2021/173

How to Cite This Article:

Kaur S, Laxmareddygari S, Gupta N, et al. Prevalence of NAFLD and its correlation with diabetic nephropathy in type 2 diabetes mellitus patients. J Evolution Med Dent Sci 2021;10(11):809-812, DOI: $10.14260 /$ jemds $/ 2021 / 173$

Submission 06-11-2020,

Peer Review 24-01-2021,

Acceptance 30-01-2021,

Published 15-03-2021.

Copyright (C) 2021 Simran Kaur et al. This is an open access article distributed under Creative Commons Attribution License [Attribution 4.0 International (CC BY 4.0)] 


\section{BACKGROUND}

Diabetes mellitus (DM) is a disorder of metabolism which is associated with hyperglycemia due to relative or absolute insulin secretion defect, varying degrees of insulin resistance or both. It is an important cause of morbidity and mortality worldwide. ${ }^{1}$ The diabetic milieu activates the reninangiotensin-aldosterone system (RAAS) and numerous other downstream mediators which play a major role in triggering the development of diseases related to kidney, by making alterations in the renal plasma flow, increasing filtration fraction, increasing kidney size which in the initial states of diabetic kidney disease increases the glomerular filtration rate abnormally. This stage is often referred to as glomerular hyperfiltration. There are also changes that affect the haemodynamics of the glomerulus, expanding oxidative stress and inducing expression of genes that favour infiltration by macrophages and cause inflammation and fibrosis in cells of vascular smooth muscle and mesangium. In addition, angiotensin II promotes proliferation of vascular smooth muscle and mesangial cells. Vascular proliferation and endothelial permeability are increased in diabetic kidney disease and are mediated by vascular endothelial growth factor (VEGF). Activated cells of inflammation, most importantly T cells, help in syntheses of angiotensin, thereby encouraging production of intrarenal angiotensin.2,3,4 Damage to the proximal tubular cell from angiotensin and albuminuria results in increased transforming growth factor (TGF)-beta with consequent conversion of pericytes into myofibroblasts and thereby resulting in excess of collagen and fibronectin deposition resulting in fibrosis.

Increased renal plasma flow and increased filtration fraction may be due to an increase in the kidney size, but they are due to the result of disproportionately reduced afferent versus efferent arteriole resistance. Increased circulating vasodilators like atrial natriuretic peptide (ANP), nitric oxide (NO) and insulin deficiency or resistance have an impact on the afferent arteriole, thereby reducing the resistance. Increased circulating vasoconstrictors like thromboxane, angiotensin II and endothelin1, in contrast, have a profound effect on the efferent arteriole thereby increasing its resistance.

The relationship of NAFLD with type 2 diabetes mellitus (T2DM) has been well studied and accepted. It can be described by resistance of insulin leading to compensatory increase in insulin progressing to defects in the metabolism of lipids and accumulation of triglycerides (TG) in the hepatic cells which ultimately leads to dysfunction of $\beta$-cells or NAFLD in T2DM. On Comparing with the non-diabetic subjects, the patients having T2DM had an increased risk to develop NAFLD and also had more risk of development of advanced liver diseases like fibrosis, cirrhosis and hepatocellular carcinoma (HCC).5,6,7 Hence the present study was carried out for assessing the prevalence of non-alcoholic fatty liver disease and its correlation with patients having diabetic nephropathy in T2DM.

NAFLD refers to accumulation of fat in hepatocytes which is otherwise known as hepatic steatosis, when all the other secondary causes like central obesity, metabolic syndrome, T2DM and heavy alcohol consumption are ruled out. It is a heterogenous disease that is influenced by multiple factors like age, ethnicity, gender and metabolic status.
NAFLD includes non-alcoholic fatty liver and NASH, i.e., non-alcoholic steatohepatitis. The key difference between NAFL and NASH is that there is no inflammation or fibrosis in the NAFL, whereas there is inflammation in the NASH. It is difficult to distinguish NASH from alcoholic hepatitis. NAFLD can progress to cirrhosis and is one of the important causes of the cryptogenic cirrhosis. The relationship between T2DM and NAFLD was established when they found elevated levels of aspartate transaminase (AST) a liver enzyme in some T2DM patients. However, the studies are still limited explaining the causal relationship between NAFLD and T2DM patients.

Routine surveillance for NAFLD in adolescents with T2DM includes abdominal examination for hepatomegaly and annual monitoring of serum aminotransferase concentrations and albuminuria for development of diabetic nephropathy. Patients who have persistent rise in aminotransferase levels and albuminuria in nephritic or nephrotic range should be evaluated further.

T2DM patients when compared with non-diabetic patients seem to have an increased risk of development of NAFLD and definitely have a higher risk of development of fibrosis due to the deposition of collagen, fibronectin and cryptogenic cirrhosis. In a study 6.5 percent of the population had mild ALT elevations shortly after the diagnosis with T2DM. In this study, 16 percent of the study population experienced more than 1.5 times rise in ALT, whereas onethird experienced 2.5 times rise in ALT. It has been estimated that approximately more than two thirds of T2DM patients may have some degree of NAFLD; but the exact prevalence of NAFLD in patients having T2DM was not known. There are a few available studies which are small and performed in highly selected populations or those studies which have estimated only the prevalence of abnormal levels of aminotransferases, which are a poor proxy measure of NAFLD.

\section{METHODS}

The present study was conducted in the Department of General Medicine from August 2019 to August 2020 and it was a comparative cross-sectional study design that included assessment of the prevalence of non-alcoholic fatty liver disease and its correlation with diabetic nephropathy in type 2 diabetes mellitus patients. Ethical clearance was obtained from the institutional ethical committee and written consent was obtained from all the patients after explaining the entire research protocol in detail. A total of 75 type 2 diabetic patients were enrolled in the present study.

Patients who didn't give the informed consent, patients with positive history of any known drug allergy and patients with presence of any malignant neoplasm were excluded from the study.

Complete demographic and clinical details of all the patients were obtained. Blood samples were obtained from all the patients and routine investigations were carried out. USG abdomen was performed in all patients after 12 hours of fasting, and fatty liver was diagnosed based on standard criteria. Diagnosis of diabetic nephropathy was done by a positive persistent albuminuria and by evaluating spot urine albumin to creatinine ratio (ACR) values of the patients. The 
prevalence of NAFLD and diabetic nephropathy was assessed among all the diabetic patients.

\section{Statistical Analysis}

All the results that were recorded during the course of this study were entered in Microsoft Excel sheet and were analysed with the help of SPSS software. Level of significance was evaluated with help of chi-square test. P-value of less than 0.05 was taken to be significant.

\section{RESULTS}

In the present study, total of 75 type 2 diabetic patients were enrolled. The mean age was $50.18 \pm 11.68$ years. Majority of the $(n=28)$ diabetic patients belonged to the age of $41-50$ years. 45 patients were females while the remaining were males (Table 1). NAFLD was found to be present in 57 patients. Therefore, 76 percent of the patients had NAFLD (Table 2). In this study, urine samples were taken to check for the presence of urinary albumin. Out of 75 patients of type 2 diabetes mellitus patients, 31 patients were positive for urine albumin. Of all the 31 diabetic patients ( $42.1 \%$ ) with positive ACR, on further ultrasound examination, 25 patients had fatty liver (Table 3). In this study, on doing urine ACR examination, 29 out of 75 diabetic patients had urine ACR value between $30-300 \mathrm{mg} / \mathrm{g}$ amongst which 15 patients (39.5\%) had fatty liver. 2 patients out of 50 diabetic patients (5.3\%) had urine ACR values $>300 \mathrm{mg} / \mathrm{g}$ and had fatty liver (Table 4).

\begin{tabular}{|c|c|c|c|c|c|c|}
\hline \multicolumn{6}{|c|}{ Parameter } & Number \\
\hline \multicolumn{6}{|c|}{ Number of patients } & 75 \\
\hline \multirow{4}{*}{\multicolumn{4}{|c|}{ Age group (years) }} & & $<=40$ & $17(22.6 \%)$ \\
\hline & & & & & $41-50$ & $28(37.3 \%)$ \\
\hline & & & & & $51-60$ & $13(17.3 \%)$ \\
\hline & & & & & $>60$ & $17(22.6 \%)$ \\
\hline \multirow{2}{*}{\multicolumn{4}{|c|}{ Gender }} & & Female & $45(60 \%)$ \\
\hline & & & & & Male & $30(40 \%)$ \\
\hline \multicolumn{7}{|c|}{ Table 1. Demographic Data of Patients of Type 2 Diabetes } \\
\hline \multicolumn{3}{|c|}{ Parameter } & \multicolumn{3}{|c|}{ Number } & Percentage \\
\hline Prevalen & of $\mathrm{N}$ & FLD & \multicolumn{3}{|c|}{57} & 76 \\
\hline \multicolumn{7}{|c|}{ Table 2. Prevalence of NAFLD } \\
\hline \multirow[b]{2}{*}{$\begin{array}{l}\text { Urine } \\
\text { Albumin }\end{array}$} & \multicolumn{3}{|c|}{ Yes $(N=57)$} & \multicolumn{2}{|c|}{$\begin{array}{c}\text { No }(N= \\
18)\end{array}$} & \multirow[b]{2}{*}{ P-Value Significance } \\
\hline & $\mathbf{N}$ & & $\%$ & \multicolumn{2}{|c|}{ F $\quad \% \quad x^{2}$ df } & \\
\hline Present & 24 & 24 & 42.1 & 0 & 0.0 & \\
\hline Absent & 51 & 33 & 57.9 & 18 & $100.05 .621 \quad 1$ & $0.018^{\$}$ \\
\hline Acc & $\begin{array}{r}\text { Ta } \\
\text { ordir }\end{array}$ & $\begin{array}{l}\text { le } 3 \\
\text { to }\end{array}$ & $\begin{array}{l}\text { Distril } \\
\text { Irine A }\end{array}$ & lbutic & $\begin{array}{l}\text { of of Diabetic } \\
\text { nin-Non-Alco }\end{array}$ & $\begin{array}{l}\text { Patients } \\
\text { holic Fatty Liver }\end{array}$ \\
\hline & & & & Yes & $N=57)$ & No $(N=18)$ \\
\hline Urine A & S Le & & $\mathbf{N}$ & $\mathbf{F}$ & $\%$ & $\%$ \\
\hline No & & & 44 & 32 & $56.1 \quad 12$ & 66.7 \\
\hline Moderatel & incre & & 29 & 23 & $40.3 \quad 6$ & 33.3 \\
\hline Severely & ncrea & & 2 & 2 & $3.5 \quad 0$ & 0.0 \\
\hline & able & $U r$ & $\overline{e A C R}$ & $\overline{\text { Leve }}$ & I - Non-Alcoho & lic Fatty Liver \\
\hline
\end{tabular}

\section{DISCUSSION}

Non-alcoholic fatty liver disease (NAFLD) is a very common disorder of metabolism and it refers to a group of conditions which lead to accumulation of excess fat in the hepatocytes of people who drink little or no alcohol and all other secondary causes like central obesity, metabolic syndrome, heavy alcohol consumption are ruled out. NAFLD has emerged worldwide as a major public health issue, with a global pooled prevalence of $25.24 \%$ by imaging, amongst general population. Maximum patients having NAFLD are in their 40s or 50s. Studies do vary regarding the distribution of sex. It is suggested by some people that NAFLD is more common in women and others suggest that it is more common in men.

NAFLD includes a spectrum of diseases which range from nonalcoholic fatty liver with fibrosis to nonalcoholic steatohepatitis (NASH), and later to advanced fibrosis with cryptogenic cirrhosis leading to hepatocellular carcinoma, which cause morbidity and mortality related to liver.7,8 NAFL refers to accumulation of fat in the liver cells, also known as hepatic steatosis without inflammation or fibrosis. NASH refers to non-alcoholic fatty liver with inflammation.

The present study was carried out for assessment of the prevalence of non-alcoholic fatty liver disease and its relationship with diabetic nephropathy in patients with type 2 diabetes mellitus. In the present study, a total of 75 type 2 diabetic patients were enrolled. Mean age of the diabetic patients was found to be 50.18 years. NAFLD was found to be present in 57 patients. Therefore, the overall prevalence of NAFLD was present in 76 percent of the patients.

The overall prevalence of diabetic nephropathy was found to be 42 percent among diabetic patients. Out of 31 patients with diabetic nephropathy, NAFLD was found to be present in 25 patients. While assessing the association of NAFLD with diabetic nephropathy in type 2 diabetic patients, significant results were obtained.

Nathalie C. Leite et al. investigated the relationship between non-alcoholic fatty liver disease (NAFLD) and diabetic nephropathy in these patients. ( $(($ The study was a cross sectional study and was conducted on one hundred and eighty patients suffering from type 2 diabetes and they were subjected to a complete clinical and laboratory evaluation and abdominal ultrasound for NAFLD detection. Fatty liver based on standard criteria was diagnosed with exclusion of significant alcohol consumption, exclusion of other causes and absence of coexisting chronic liver disease, and bright liver because of diffuse fatty infiltration, contrast between the echogenicity of the liver with the fatty deposit showing hyper echogenicity, the blood vessels and kidneys fading rate. Screening for micro albuminuria in urine was done by the most used method, measurement of the spot urine albuminto-creatinine (UACR) ratio in a random collection. 180 patients having T2DM were taken in this study, out of which $58(32 \%)$ patients were males and $122(68 \%)$ patients were females. Of these 156 patients (86.66\%) had non-alcoholic fatty liver disease and diabetic nephropathy was seen amongst $35 \%$ of subjects. ${ }^{9}$

Heidari $\mathrm{Z}$ et al. investigated the association between nonalcoholic fatty liver disease (NAFLD) and diabetic nephropathy in 255 patients. A cross sectional study was conducted on 255 patients with T2DM with a minimum age of 30 years. Fatty liver was diagnosed in patients who consumed very little or no alcohol, and fatty infiltration was seen on ultrasonography, with mildly deranged liver enzymes and urine albumin to creatinine ratio showing microalbuminuria. 255 patients with diagnosed T2DM were 
enrolled in this study, out of which 82 (32\%) were males and $173(68 \%)$ were females. Out of 255 patients, 221 patients (86.66 \%) had NAFLD and diabetic nephropathy was seen in $33 \%$ of subjects, microalbuminuria was seen amongst $32 \%$ of subjects and macroalbuminuria was seen in $10 \%$ of all individuals. ${ }^{10}$

In a study conducted by G Targher et al., they concluded that NAFLD is associated with increased risk of diabetic nephropathy independent of several other cofounding factors. ${ }^{11}$ In a study conducted by Laltesh Kumar et al., in Varun Medical college of Uttar Pradesh, they found that NALFD was not a significant risk factor in the development of diabetic nephropathy. ${ }^{12}$

In a study conducted by Guoyu et al., they concluded that NAFLD might be an important risk factor which can lead to the development of diabetic nephropathy because of the burden of the elevated fat content. ${ }^{13}$ In a study conducted by Yasui et al., they concluded that diabetic nephropathy was present in 14 percent of the patients with NAFLD. ${ }^{14}$

In a study conducted by Yilmaz $\mathrm{Y}$ et al., they concluded that microalbuminuria was present in 16 percent of the patients with NAFLD. ${ }^{15}$ In a study conducted by Sinn DH et al., they found out that the incidence of diabetic nephropathy was significantly higher in patients with NAFLD. ${ }^{16}$

In a study conducted by Kasapoglu B et al., they found out that patients with NAFLD had significantly greater albumin creatinine ratio ${ }^{17}$ than those without. In a study by Yeung MW et al., type 2 diabetes mellitus patients with NASH had significant albuminuria than those patients without NASH. ${ }^{18}$

In a study by Hwang ST et al., NAFLD patients had significantly higher prevalence rates of microalbuminuria and significantly greater albumin creatinine ratio $^{19}$ in $\mathrm{T} 2 \mathrm{DM}$ patients than those without T2DM.

\section{CONCLUSIONS}

NAFLD is a major risk factor for the development of diabetic nephropathy in patients diagnosed with type 2 diabetes mellitus.

Data sharing statement provided by the authors is available with the full text of this article at jemds.com.

Financial or other competing interests: None.

Disclosure forms provided by the authors are available with the full text of this article at jemds.com.

\section{REFERENCES}

[1] Lutale JJK, Thordarson H, Abbas ZG, et al. Microalbuminuria among type 1 and type 2 diabetic patients of African origin in Tanzania. BMC Nephrol 2007;8:2.

[2] Ritz E. Nephropathy in type 2 diabetes. J Intern Med 1999;245(2):111-26.

[3] Hatfield P, Hay N, Macdonald A, et al. Nephropathy in diabetes. N Z Med J 1997;110(1036):19.

[4] Battisti WP, Palmisano J, Keane WF. Dyslipidemia in patients with type 2 diabetes. Relationships between lipids, kidney disease and cardiovascular disease. Clin Chem Lab Med 2003;41(9):1174-81.

[5] Younossi ZM, Gramlich T, Christi A. et al. Type 2 diabetes. Clin Gastroenterol Hepatol 2004;2:262-5.

[6] Targher G, Bertolini L, Rodella S, et al. Nonalcoholic fatty liver disease is independently associated with an increased incidence of cardiovascular events in type 2 diabetic patients. Diabetes Care 2007;30(8):2119-21.

[7] Parving HH, Gall MA, Skøtt P, et al. Prevalence and causes of albuminuria in non-insulin-dependent diabetic patients. Kidney Int 1992;41(4):758-62.

[8] Targher G, Bartolini L, Rodella S, et al. Non-alcoholic fatty liver disease is independently associated with an increased prevalence of chronic kidney disease in type 2 diabetic patients. Diabetologia 2008;51(3):444-50.

[9] Leite NC, Salles GF, Araujo ALE, et al. Prevalence and associated factors of non-alcoholic fatty liver disease in patients with type-2 diabetes mellitus. Liver Int 2009;29(1):113-9.

[10] Article O, Heidari Z, Gharebaghi A. Prevalence of non alcoholic fatty liver disease and its association with diabetic nephropathy in patients with type 2 diabetes mellitus. J Clin Diagnostic Res 2017;11(5):OC4-OC7.

[11] Section of Endocrinology and Metabolism. Department of Biomedical and Surgical Sciences, University of Verona, Ospedale Civile Maggiore, Piazzale Stefani 1, 37126, Verona, Italy. giovanni.targher@univr.it

[12] Kumar L, Kumar A. Prevalence of non-alcoholic fatty liver disease and its association with diabetic nephropathy in type 2 diabetes mellitus patients. International Journal of Contemporary Medicine Surgery and Radiology 2020;5(1):A243-A6.

[13] Jia G, Di F, Wang Q, et al. Non-alcoholic fatty liver disease is a risk factor for the development of diabetic nephropathy in patients with type 2 diabetes mellitus. PLoS One 2015;10(11):e0142808.

[14] Yasui K, Sumida Y, Mori Y, et al. Nonalcoholic steatohepatitis and increased risk of chronic kidney disease. Metabolism 2011;60(5):735-9.

[15] Yilmaz Y, Alahdab YO, Yonal O, et al. Microalbuminuria in nondiabetic patients with nonalcoholic fatty liver disease: association with liver fibrosis. Metabolism 2010;59(9):1327-30.

[16] Sinn DH, Kang D, Jang HR, et al. Development of chronic kidney disease in patients with non-alcoholic fatty liver disease: a cohort study. J Hepatol 2017;67(6):1274-80.

[17] Kasapoglu B, Turkay C, Yalcın KS, et al. Increased microalbuminuria prevalence among patients with nonalcoholic fatty liver disease. Ren Fail 2016;38(1):159.

[18] Yeung MW, Wong GLH, Choi KC, et al. Advanced liver fibrosis but not steatosis is independently associated with albuminuria in Chinese patients with type 2 diabetes. J Hepatol 2018;68(1):147-56.

[19] Hwang ST, Cho YK, Yun JW, et al. Impact of non-alcoholic fatty liver disease on microalbuminuria in patients with prediabetes and diabetes. Intern Med J 2010;40(6):43742. 